\title{
CD review
}

\section{Perfect posture for the dental team P. Ellis and Smile-on Ltd \\ Smile-on Ltd \\ $\mathfrak{E} 199.00$ + VAT}

Those of us who are familiar with Smile-on Ltd's CD-ROM based e-learning packages for the dental team will recognise the house style' of this CD immediately.After DNNART and DNNET (learning packages for dental nurses) they have made this type of computer based education their own.

This time the two hour course is concerned with improving posture and practising four-handed dentistry for the whole clinical team.

Its strap-line is 'better posture is equal to less pain which leads to less stress in the surgery'. To see if this worked we put the CD-ROM to several different uses in our dental practice. It was viewed by:

- An experienced dentist and nurse team as a tool for revision

- A dental nurse who had recently joined the practice and had never experienced four-handed dentistry before, as an introduction to the subject

- A dental nurse tutor, to assess its suitability as a training tool for all team members.

For those who do not always like to follow the instructions on-screen, there is an accompanying booklet which explains how to get the most out of this course.

It is split up into different sections covering aspiration, soft tissue retraction, instrument handling and transfer and clinical procedure examples. It aims to address the following:

- Preventing back and neck pain

- Reducing work stress

- Improving access and vision

- Working more effectively as a team.

The CD begins with a visit by the user to 'The Posture Practice.' By clicking on the various individuals you can learn about the problems of a dental nurse, dentist and hygienist with regard to their back problems due to poor posture in the surgery. The dentist character tells how he was thinking of taking early retirement due to his pain brought on by bending over patients for many years. He says as a result of watching the programme he has learnt 'to use his nurse more effectively'! I think I would have had more sympathy with him if he had said something along the lines of 'working together more efficiently as a team'.

Moving though the various sections there are plenty of opportunities to interact with what is happening on the screen. Using the 'drag and drop' action in the section on instrument exchange you are asked to make choices on correct use of the transfer zone and various sequence of use for the instruments.

A useful addition is the opportunity to access a PDF file to print out exercises to try in your own surgery. We tried this option, stopping the $\mathrm{CD}$, and as a team putting into practice what we had just seen on-screen. This definitely worked. When we were unsure of an instrument transfer or spotted a posture fault we made a note and then revisited the relevant section on the $\mathrm{CD}$.

The programme is a good introduction to what is in my opinion a neglected subject. I have always felt that if a dental nurse is uncomfortable, and unable to see clearly what she is doing at the chairside, she is unlikely to be able to anticipate the dentist's next move and offer useful assistance.

There were only a few things that we didn't like about this CD. The role of dental dam was not emphasised enough. There is a whole section on tissue retraction. If dental dam was used more in operative procedures there would be no real need for the dental nurse to put huge effort into holding back the cheeks and depressing the tongue just to give her access. With dam in place the field of vision is near perfect.

The dental nurse is strongly encouraged to rinse the patient's mouth out using the 3:1 syringe and suction thus eliminating the need for a conventional rinse. Rinsing, they say, breaks the concentration of the team, and the patient fails to locate the exact position they had in the chair prior to rinsing. We (the team) say letting the patient sit up occasionally gives the team a tiny break and the patient too no doubt!

Using this type of e-learning - where at the end of the programme we each printed

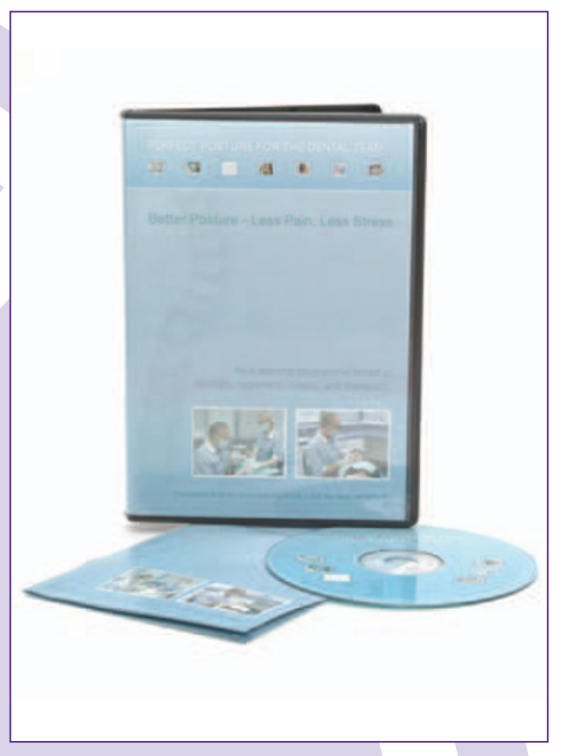

out a certificate worth two hours of verifiable CPD having never left the comfort of our own practice - makes me wonder how we managed without it! By stopping the CD when we liked and going into our own surgery to try out the theory straight away, I feel we are more likely to put the learning into practice. When you attend a formal course so much knowledge and enthusiasm is lost between the end of the lecture and the next time you are in the surgery. We can also get more mileage out of this $\mathrm{CD}$ when we need revision or a new team member joins the practice.

This CD might seem expensive, but it's only when you start to consider the cost of taking the team to a 'hands-on' course at a distant location plus travel that you start to realise how realistic the price really is.

At the risk of sounding clichéd, the CD 'does what is says on the package'. Better posture $=$ less pain and less stress. Bring it on!

\section{Kathryn Marshall RDN Dip DHE}

Nurse in dental practice, Oxted, Surrey

Clinical Dental Lead (DCPs) Kent, Surrey \& Sussex Deanery

Practice Development Tutor, School of

Postgraduate Medical \& Dental

Education Wales

Read more about four-handed dentistry on page 34. 